\title{
Acetylcholinesterase inhibitory potencies of new pyrazoline derivatives
}

\author{
Mehtap TUĞRAK ${ }^{1}$ (D), Halise İnci GÜL ${ }^{*}$ (D), İlhami GÜLÇİN 2 (D) \\ 1 Department of Pharmaceutical Chemistry, Faculty of Pharmacy, Ataturk University, Erzurum, Turkey. \\ 2 Department of Chemistry, Faculty of Science, Ataturk University, Erzurum, Turkey \\ * Corresponding Author. E-mail: incigul1967@yahoo.com (H.İ.G.); Tel. +90-0442-231 5219.
}

Received: 19 February 2020 / Revised: 23 April 2020 / Accepted: 28 May 2020

ABSTRACT: Alzheimer's disease (AD) has no current cure and its mechanism is not fully known, but treatments for symptoms are available. Acetylcholinesterase (AChE) has been reported to be an applicable therapeutic target in patient with AD. Acetylcholinesterase inhibitors (AChEIs) are commonly used for it. For this purpose, novel series of pyrazoline based compounds [2-(3-(4-methoxyphenyl)-5-aryl-4,5-dihydro-1H-pyrazol-1-yl)benzo[d]thiazole, 1-9] were synthesized and $\mathrm{AChE}$ inhibitory potencies were reported here. The results indicated that compound 1 (Ki= $0.13 \pm 0.004 \mu \mathrm{M})$ possessed the highest $\mathrm{AChE}$ inhibitory effect in series, which is two times more potent than the reference compound Tacrin $(\mathrm{Ki}=0.26 \pm 0.045 \mu \mathrm{M})$. So, pyrazoline derivative 1 can be considered as a lead inhibitor in designing new AChE inhibitors.

KEYWORDS: Acetylcholinesterase; alzheimer disease; benzothiazol; pyrazoline.

\section{INTRODUCTION}

Alzheimer's disease (AD) is a destructive brain disorder among elderly people. The first of the pathophysiological factors associated with $\mathrm{AD}$ is the collection of $\beta$-amyloid protein exterior to the neurons in the encephalon, another is the collection of twisted tau protein (tangles) strands interior of the neurons. In addition, AD patients undergo significant loss of neurons especially in the hippocampus. Many neurotransmitter levels are significantly reduced in this disease. These are serotonin, dopamine, glutamate, noradrenaline and acetylcholine (ACh). Decreased neurotransmitter levels also cause memory, cognition, motor and functional capacity loss. This disease often affects people who are exposed to stress. The number of people suffering from this disease will certainly increase in future due to aging population, insufficient personal care, deficient health support for patients and their families [1-4].

$\mathrm{ACh}$ is a critical neurotransmitter in the brain, which is especially important for attention, memory and motivation. Studies have shown that this neurotransmitter decreases dramatically in the brain cortex and hippocampus of Alzheimer's patients. The cholinergic supposal proposes that molecules that goal and prevent the acetylcholinesterase enzyme (AChE) will be useful for the treatment of $\mathrm{AD}$ for instance rivastigmine, galantamine, and donepezil (Figure 1). They cause increasing of ACh concentration by inhibiting AChE enzyme and prevent acetylcholine (ACh) hydrolysis. Although neurotransmitter therapy causes improvements in cognitive skills, such improvements are usually temporary. The drugs used in therapy aim to reduce $\mathrm{AD}$ symptoms rather than disease progression. Therefore, the design and development of new compounds that may be acetylcholineesterase inhibitors are important and good choice in the treatment of $\mathrm{AD}$ and development of new drug candidates for $\mathrm{AD}$ [3-5] .

2-Pyrazolines are most popular heterocyclic structure in drug design. They have various important biological activities such as as antimicrobial [6], antifungal [7], antimalarial [8], antiinflammatory [9-10], analgesic [10], anti-Alzheimer's disease [11], anti-Parkinson's disease [12], antidepressant [13-14], anticancer [15-16] and monoamine oxidase (MAO) inhibitory activities [17-18]. In recent years, pyrazoline derivatives are shown to have inhibiting or activating effects on several enzymes such as cholinesterases [19], MAOs, and carbonic anhydrases [20-21].

How to cite this article: Tuğrak M, Gül Hi, Gülçin I. Acetylcholinesterase Inhibitory Potency of New Pyrazoline Derivatives J Res Pharm. 2020; 24(4): 464-471. 
<smiles>COc1cc2c(cc1OC)C(=O)C(CC1CCN(Cc3ccccc3)CC1)C2</smiles>

Donepezil<smiles>CCN(C)C(=O)Oc1cccc(C(C)N(C)C)c1</smiles>

Rivastigmine

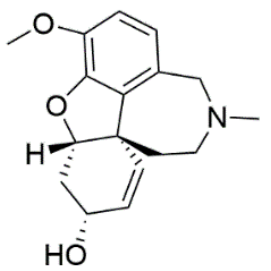

Galantamine

Figure 1. Drugs commonly prescribed for Alzheimer's disease.

In addition, benzothiazole, the subject of this research, was considered as an important pharmacophoric group because of its wide range of bioactivities such as antifungal activity [22], carbonic anhydrase inhibition [23], antihypoxic [24] and monoamine oxidase type A (MAO-A) inhibition [25].

It was aimed to synthesize new 2-(3-(4-methoxyphenyl)-5-aryl-4,5-dihydro-1H-pyrazol-1yl)benzo[d]thiazoles (1-9) to investigate their AChE inhibitory effects to ascertain possible lead compounds in this study.

\section{RESULTS AND DISCUSSION}

\subsection{Chemistry}

2-(3-(4-Methoxyphenyl)-5-aryl-4,5-dihydropyrazol-1-yl)benzo[d]thiazoles $\mathbf{1 - 9}$ were synthesized succesfully as presented in Figure 2. In the study, phenyl (1), 4-methylphenyl (2), 4-chlorophenyl (3), 4fluorophenyl (4), 4-bromophenyl (5), 3-bromophenyl (6), 2-bromophenyl (7), 2-chlorophenyl (8), 2,6dichlorophenyl (9) were used as aryl part. Compounds synthesized are original, except $\mathbf{1}$ and 3.

When the ${ }^{1} \mathrm{H}$ NMR spectra were examined, the proton of the C-5 carbon of the pyrazoline ring resonated as a doublet of doublet between the range of 6.40-5.61 ppm. The other protons on C-4 resonated as a doublet of the doublet. One of these protons resonated in the range of 4.01-3.95 ppm, while the other proton resonated in the range of 3.15-3.46 ppm. Both protons were found to have two J values.

One of the protons of the pyrazoline ring of the many compounds was coincided with the methoxy peak existed in the main structure. It was observed that the protons belonging to the methoxy group had a resonance in the range of 3.87-3.78 ppm.

It has been observed that C-4, C-5 and methoxy groups of the pyrazoline ring resonated in the range of 63.6-43.2 ppm in the ${ }^{13} \mathrm{C}$ NMR. Chemical shift values of compounds $(\mathbf{2}, \mathbf{4}-\mathbf{9})$ were presented for the first time in detail in the experimental section. These values showed that the synthesized compounds were coherent with their chemical structure.

\subsection{Acetylcholinesterase inhibitory activity}

Recombinant human AChE enzyme was used to evaluate AChE inhibitory activity of the compounds [20]. Tacrine (TAC) was utilized as a reference drug. Table 1 shows that the compounds had $\mathrm{IC}_{50}=1.30-2.78$ $\mu \mathrm{M}$ and $\mathrm{Ki}=0.13 \pm 0.004-0.85 \pm 0.070 \mu \mathrm{M}$ values. In addition, reference compound $\mathrm{TAC}$ had value of $\mathrm{IC}_{50}=0.84$ $\mu \mathrm{M}$ and $\mathrm{Ki}=0.26 \pm 0.045 \mu \mathrm{M}$.

When the effect of the groups on the phenyl ring was evaluated in terms of $\mathrm{IC}_{50}$, both the electron donating group (compound 2 which had $4-\mathrm{CH}_{3}$ substituent, $\mathrm{IC}_{50}=2.49 \mu \mathrm{M}$ ) and the electron withdrawing groups (compound 3 which had $4-\mathrm{Cl}$ substituent, $\mathrm{IC}_{50}=2.43 \mu \mathrm{M}$, and compound 5 which had $4-\mathrm{Br}$ substituent, $\mathrm{IC}_{50}=2.45 \mu \mathrm{M}$ ) in para position of phenyl ring reduced the inhibitory potency compared to the unsubstituted phenyl analog $1\left(\mathrm{IC}_{50}=1.30 \mu \mathrm{M}\right)$. When para position halogenated compounds 3, 4, $5\left(\mathrm{IC}_{50}=\right.$ $2.43,1.48,2.45 \mu \mathrm{M}$, respectively) were compared, compound 4 had the strongest inhibitory effect among others according to its $\mathrm{IC}_{50}$ value. On the other hand, compound 5 has shown the lowest inhibitory property. The reason that compound 5 with bromine at 4 -th position has the lowest inhibitory property among the 
halogenated ones may be due to the bigger atomic diameter of bromine than chlorine and fluorine atoms. On the other hand, when the effect of position of bromine substitution was compared, 4-bromo derivative compound 5, 3-bromo derivative compound 6, and 2-bromo derivative compound 7; compound $\mathbf{6}$ showed great $\mathrm{AChE}$ inhibitory potency than other brominated ones with $\mathrm{IC}_{50}$ value of $1.42 \mu \mathrm{M}$.

Compound 1 displayed the best $\mathrm{AChE}$ inhibition potency with $\mathrm{IC}_{50}$ value of $1.30 \mu \mathrm{M}$ among others. When chlorine substituent (compound 3, 8 and 9) at different positions of the phenyl ring was evaluated in terms of $\mathrm{IC}_{50}$, compound $\mathbf{8}$, which has chlorine at orto position, had the strongest inhibitory effect among others. The reason for lower inhibition observed in other derivatives ( $\mathbf{3}$ and $\mathbf{9}$ ) may be that compound 8 might have interacted with different active sites of the enzyme.

Majority of the compounds evinced notable inhibition properties toward AChE enzyme in micromolar concentrations. The compound $\mathbf{1}$ was ascertained preeminent AChE inhibitor with the lowest Ki value of $0.13 \pm 0.004 \mu \mathrm{M}$. In addition, compound 1 exhibited better inhibition profile toward AChE than Tacrine. Furthermore, the halogen size has been found to be considerable in reducing the inhibition potential on AChE. Compound 4 (with fluorine) showed more potent inhibitory activity than other halogenated ones with Ki value of $0.19 \pm 0.056 \mu \mathrm{M}$.

Table 1. Inhibitory effects of pyrazoline derivatives 1-9 on AChE.

\begin{tabular}{cccc}
\hline & $\mathrm{IC}_{50}(\mu \mathrm{M})$ & & $\mathrm{Ki}(\mu \mathrm{M})$ \\
\hline Compounds & $\mathrm{AChE}$ & $\mathbf{r}^{\mathbf{2}}$ & $\mathrm{AChE}$ \\
\hline $\mathbf{1}$ & 1.3 & 0.9236 & $0.13 \pm 0.004$ \\
$\mathbf{2}$ & 2.49 & 0.9889 & $0.52 \pm 0.560$ \\
$\mathbf{3}$ & 2.43 & 0.9846 & $0.29 \pm 0.006$ \\
$\mathbf{4}$ & 1.48 & 0.944 & $0.19 \pm 0.056$ \\
$\mathbf{5}$ & 2.45 & 0.9863 & $0.68 \pm 0.137$ \\
$\mathbf{6}$ & 1.42 & 0.9646 & $0.53 \pm 0.058$ \\
$\mathbf{7}$ & 1.88 & 0.9518 & $0.85 \pm 0.070$ \\
$\mathbf{8}$ & 2.00 & 0.972 & $0.50 \pm 0.055$ \\
$\mathbf{9}$ & 2.78 & 0.9861 & $0.51 \pm 0.054$ \\
TACa & 0.84 & 0.9844 & $0.26 \pm 0.045$
\end{tabular}

aTacrine (TAC) was used as a standard inhibitor for $\mathrm{AChE}$. $\mathrm{IC}_{50}$ value is the half maximal inhibitory concentration of inhibitors used for enzyme inhibition. $\mathrm{Ki}$ is the inhibition constant and the dissociation equilibrium constant of the enzyme-inhibitor complex. $r$-squared $\left(\mathrm{r}^{2}\right)$ value presents a variety of goodness-of-fit statistics.

When the $\mathrm{Ki}$ results of the compounds were evaluated, nonsubstituted compound $\mathbf{1}$ has drawn attraction in series. When substition effects of the groups on the phenyl ring was taken into account, methyl substitution at compound 2, which is a electron releasing group, inhibition of AChE by 2 decreased 4.17 times comparing to $\mathbf{1}$, which is a nonsubstituted compound. However, halogenated compound 3 (with chlorine), 4 (with fluorine), 5 (with bromine) were considered in terms of their Ki value; fluorinated compound 4 was found more effective on AChE than the other two halogenated compounds 3 and 5 . This situation most probably results from the small size of fluorine atom than chlorine and bromine atoms. In this study, halogenated compounds (compounds 5, 6, 7), which have halogen atom of different position of phenyl ring, were synthesized. The effect of bromine position at the compound 5, 6, and 7 was also evaluated. By considering Ki values of them, in case of compound 6, which has bromine at the 3rd position of phenyl, was seen as the most potent inhibitor among compounds 5, 6, and 7. In addition, the same situation was evaluated for the compounds 3,8 and $\mathbf{9}$. In this case, compound 3 , which has chlorine at the 4th position of phenyl ring, was found as the most effective compound while compound 8, which is 2-chloro substituted and compound 9, which is 2,6-dichloro substituted, have shown similar inhibition property in series. According to Ki values, compound $\mathbf{1}$ has been found as the most effective compound of series. 


\section{CONCLUSION}

This study reported synthesis and AChE inhitory potency of the new pyrazoline derivatives (1-9). The results indicated that compound $\mathbf{1}$ had the greatest inhibitory potency with both $\mathrm{IC}_{50}(1.30 \mu \mathrm{M})$ and $\mathrm{Ki}$ $(0.13 \pm 0.004 \mu \mathrm{M})$ values. Based on the results of enzyme inhibition, the pyrazoline derivative compound 1 can be taken into consideration as a candidate compound to improve new AChE inhibitors.

\section{MATERIALS AND METHODS}

\subsection{Chemistry}

During the synthesis studies, to monitor of the reaction and check the purity of the synthesized compounds, Thin Layer Chromatography (TLC) plates (60 HF254, Merck KGaA) were used. Chloroform: methanol (4.8:0.2) was used as mobile phase system. Nuclear Magnetic Resonance (NMR) spectra of the compounds were taken with the Varian Mercury Plus spectrometer (Varian inc., Palo Alto, California, U.S.). Shimadzu's LCMS-TOF-ESI (Shimadzu, Kyoto, Japan) device was used for HRMS. Electrothermal 9100/IA9100 instrument (Bibby Scientific Limited, Staffordshire, UK) was used to determine melting points.

\subsubsection{Synthesis of 2-hydrazinylbenzo[d]thiazole (Compound A, Figure 2)}

The starting compound 2-mercaptobenzothiazole ( $3.0 \mathrm{~g})$ and hydrazine hydrate $(10 \mathrm{ml} / 80 \%)$ were refluxed for 24 hours by conventional method in ethanol $(20 \mathrm{ml})$. At the end of the specified period, the contents of the flask were kept at room temperature and the separated product was filtered and dried. Light brown compound A was used for the next reaction without purification [26].

\subsubsection{General procedure for the preperation of chalcones, 1a-9a, Figure 2}

Starting compounds of the series, which are chalcones, were synthesized by Claisen-Schmidt condensation [27-31]. 4-Methoxy acetophenone and the appropriate aldehyde derivative [benzaldehyde (1a), 4-methylbenzaldehyde (2a), 4-chlorobenzaldehyde (3a), 4-fluorobenzaldehyde (4a), 4bromobenzaldehyde (5a), 3-bromobenzaldehyde (6a), 2-bromobenzaldehyde (7a), 2-chlorobenzaldehyde (8a), 2,6-dichlorobenzaldehyde (9a)] were mixed within ethyl alcohol $(6 \mathrm{ml})$ in 1: 1 mole ratio. The mixture was cooled on ice bath afterward aq. $\mathrm{NaOH}$ solution $(6 \mathrm{ml}, 10 \%)$ was added drop by drop to the flask. The mixing was sustained at room temperature throughout the night. After 24 hours, the content was taken into the cold water $(50 \mathrm{ml})$. The contents of the flask were acidified with concentrated $\mathrm{HCl}$ acid $(\mathrm{pH}=6-7)$. The collapsed solid was filtered and washed with water and ethanol. After the intermediate compound was dried, it was used as a starting material in the third step.

\subsubsection{Synthesis of the pyrazoline type compounds 1-9, Figure 2}

Synthesis of pyrazoline derivative compounds (1-9) was carried out in acidic medium using a conventional method with a protic solvent. Briefly, favorable chalcone derivative $(1 \mathrm{mmol})(\mathbf{1 a - 9})$ and 2hydrazinylbenzo[d]thiazole $(1.1 \mathrm{mmol})$ in ethanol $(25 \mathrm{~mL})$ with acetic acid $(0.05 \mathrm{~mL})$ were heated for $19-36 \mathrm{~h}$ (for 1-9) [20-21].Then, ethanol was evaporated until half volume and the flask was left at room temperature. After the collapsed solid was filtered, it was purified by crystallization from the suitable solvent or solvent mixture (methanol or methanol-ether). ${ }^{1} \mathrm{H}$ NMR, ${ }^{13} \mathrm{C}$ NMR, and HRMS were used to confirm their chemical strutures.

\section{2-(3-(4-Methoxyphenyl)-5-phenyl-4,5-dihydro-1H-pyrazol-1-yl)benzo[d] thiazole (1)}

A bright green solid, yield 9.3\%. Mp: 188-190 ${ }^{\circ} \mathrm{C}$; Lit m.p: $149-150{ }^{\circ} \mathrm{C}[32] .{ }^{1} \mathrm{H}$ NMR $\left(400 \mathrm{MHz}, \mathrm{CDCl}_{3}, \delta\right.$ ppm): 7.71 (d, 2H, Ar-H, J= 8.8 Hz), $7.63(\mathrm{~d}, 1 \mathrm{H}, \mathrm{Ar}-\mathrm{H}, J=7.7 \mathrm{~Hz}), 7.50(\mathrm{~d}, 1 \mathrm{H}, \mathrm{Ar}-\mathrm{H}, J=8.1 \mathrm{~Hz}), 7.46-7.22(\mathrm{~m}$, $6 \mathrm{H}, \mathrm{Ar}-\mathrm{H}), 7.07(\mathrm{t}, 1 \mathrm{H}, \mathrm{Ar}-\mathrm{H}, J=7.5 \mathrm{~Hz}), 6.94(\mathrm{~d}, 2 \mathrm{H}, \mathrm{Ar}-\mathrm{H}, J=8.8 \mathrm{~Hz}), 5.78(\mathrm{dd}, 1 \mathrm{H}$, pyrazoline ring, $J=12.1$, $5.1 \mathrm{~Hz}), 3.84\left(\mathrm{~s}, 3 \mathrm{H}, \mathrm{OCH}_{3}\right), 3.27(\mathrm{dd}, 1 \mathrm{H}$, pyrazoline ring, $J=17.2,5.1 \mathrm{~Hz})$, (one of the proton peak of the pyrazoline ring was under methoxy peak). ${ }^{13} \mathrm{C} \mathrm{NMR}\left(100 \mathrm{MHz}, \mathrm{CDCl}_{3}, \delta \mathrm{ppm}\right): 152.9,152.7,141.6,131.9$, 129.1, 128.7, 128.4, 127.9, 126.2, 125.8, 124.1, 121.8, 121.4, 120.9, 120.2, 114.4, 63.7, 55.6, 44.1, HRMS (ESI-MS) calc. for $\mathrm{C}_{23} \mathrm{H}_{19} \mathrm{~N}_{3} \mathrm{OS}[\mathrm{M}+\mathrm{H}]^{+}$386.1322; found 386.1336. 


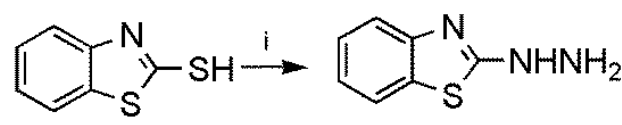

\section{Compound A}<smiles>CC=CC(=O)Br</smiles><smiles>CC#CCNNc1nc2ccccc2s1</smiles>

(1a-9a)

\section{Compound A}<smiles>COc1ccc(C2=NN(c3nc4ccccc4s3)C(Br)C2)cc1</smiles>

(1-9)

Reagents: i: Hydrazine hydrate (\%80), ethanol, reflux, ii: $\mathrm{NaOH}(\% 10)$, ethanol, rt, iii: Ethanol, glacial acetic acid, reflux. Ar: Phenyl (1), 4-methylphenyl (2), 4-chlorophenyl (3), 4-fluorophenyl (4), 4-bromophenyl (5), 3-bromophenyl (6), 2bromophenyl (7), 2-chlorophenyl (8), 2,6-dichlorophenyl (9).

Figure 2. Synthesis of compounds 1-9.

\section{2-(3-(4-Methoxyphenyl)-5-(p-tolyl)-4,5-dihydro-1H-pyrazol-1-yl)benzo[d]thiazole (2)}

A bright yellow solid, yield 11.3\%. Mp: 200-201 ${ }^{\circ} \mathrm{C} .{ }^{1} \mathrm{H}$ NMR $\left(400 \mathrm{MHz}, \mathrm{CDCl}_{3}, \delta \mathrm{ppm}\right): 7.71$ (d, 2H, Ar-H, $J=8.8 \mathrm{~Hz}), 7.62(\mathrm{~d}, 1 \mathrm{H}, \mathrm{Ar}-\mathrm{H}, J=8.1 \mathrm{~Hz}), 7.50(\mathrm{~d}, 1 \mathrm{H}, \mathrm{Ar}-\mathrm{H}, J=8.1 \mathrm{~Hz}), 7.25-7.21(\mathrm{~m}, 3 \mathrm{H}, \mathrm{Ar}-\mathrm{H}), 7.12$ $(\mathrm{d}, 2 \mathrm{H}, \mathrm{Ar}-\mathrm{H}, J=8.1 \mathrm{~Hz}), 7.06(\mathrm{t}, 1 \mathrm{H}, \mathrm{Ar}-\mathrm{H}, J=7.5 \mathrm{~Hz}), 6.94(\mathrm{~d}, 2 \mathrm{H}, \mathrm{Ar}-\mathrm{H}, J=8.8 \mathrm{~Hz}), 5.74(\mathrm{dd}, 1 \mathrm{H}$, pyrazoline ring, $J=11.7,5.0 \mathrm{~Hz}), 3.85\left(\mathrm{~s}, 3 \mathrm{H}, \mathrm{OCH}_{3}\right), 3.26(\mathrm{dd}, 1 \mathrm{H}$, pyrazoline ring, $J=17.2,5.0 \mathrm{~Hz}), 2.30(\mathrm{~s}$, $3 \mathrm{H}, \mathrm{CH}_{3}$ ), (one of the proton peak of the pyrazoline ring was under methoxy peak). ${ }^{13} \mathrm{C} \mathrm{NMR}(100 \mathrm{MHz}$, $\mathrm{CDCl}_{3}, \delta$ ppm): 163.6, 161.4, 152.9, 152.6, 138.7, 137.6, 131.9, 129.7, 128.3, 126.2, 125.7, 124.2, 121.8, 120.9, 120.2, 114.4, 63.5, 55.6, 44.1, 21.3, HRMS (ESI-MS) calc. for $\mathrm{C}_{24} \mathrm{H}_{21} \mathrm{~N}_{3} \mathrm{OS}[\mathrm{M}+\mathrm{H}]^{+} 400.1478$; found 400.1487 .

2-(5-(4-Chlorophenyl)-3-(4-methoxyphenyl)-4,5-dihydro-1H-pyrazol-1-yl)benzo[d]thiazole (3)

A cream colour solid, yield 8.4\%. Mp: 176-177 ${ }^{\circ} \mathrm{C}$; Lit m.p:128-129 [32]. ${ }^{1} \mathrm{H}$ NMR $\left(400 \mathrm{MHz}, \mathrm{CDCl}_{3}, \delta\right.$ ppm): $7.70(\mathrm{~d}, 2 \mathrm{H}, \mathrm{Ar}-\mathrm{H}, J=8.8 \mathrm{~Hz}), 7.64(\mathrm{~d}, 1 \mathrm{H}, \mathrm{Ar}-\mathrm{H}, J=8.1 \mathrm{~Hz}), 7.50(\mathrm{~d}, 1 \mathrm{H}, \mathrm{Ar}-\mathrm{H}, J=8.1 \mathrm{~Hz}), 7.28-7.23(\mathrm{~m}$, $5 \mathrm{H}, \mathrm{Ar}-\mathrm{H}), 7.09(\mathrm{t}, 1 \mathrm{H}, \mathrm{Ar}-\mathrm{H}, J=8.1 \mathrm{~Hz}), 6.94(\mathrm{~d}, 2 \mathrm{H}, \mathrm{Ar}-\mathrm{H}, J=8.8 \mathrm{~Hz}), 5.71(\mathrm{dd}, 1 \mathrm{H}$, pyrazoline ring, $J=12.1$, $5.5 \mathrm{~Hz}), 3.85\left(\mathrm{~s}, 3 \mathrm{H}, \mathrm{OCH}_{3}\right), 3.22(\mathrm{dd}, 1 \mathrm{H}$, pyrazoline ring, $J=17.6,5.5 \mathrm{~Hz})$, (one of the proton peak of the pyrazoline ring was under methoxy peak). ${ }^{13} \mathrm{C} \mathrm{NMR}\left(100 \mathrm{MHz}, \mathrm{CDCl}_{3}, \delta \mathrm{ppm}\right)$ : 163.5, 161.5, 152.8, 152.6, 140.2, 133.7, 131.9, 129.3, 128.4, 127.8, 125.9, 123.9, 122.0, 121.0, 120.2, 114.4, 63.2, 55.6, 43.9, HRMS (ESI-MS) calc. for $\mathrm{C}_{23} \mathrm{H}_{18} \mathrm{~N}_{3} \mathrm{OSCl}[\mathrm{M}+\mathrm{H}]^{+} 420.0932$; found 420.0936 .

\section{2-(5-(4-Fluorophenyl)-3-(4-methoxyphenyl)-4,5-dihydro-1H-pyrazol-1-yl)benzo[d] thiazole (4)}

A dark yellow solid, yield 6.4\%. Mp: 163-165 ${ }^{\circ} \mathrm{C} .{ }^{1} \mathrm{H}$ NMR (400 MHz, $\mathrm{CDCl}_{3}, \delta$ ppm): 7.71 (d, $2 \mathrm{H}, \mathrm{Ar}-$ $\mathrm{H}, J=8.8 \mathrm{~Hz}), 7.63(\mathrm{~d}, 1 \mathrm{H}, \mathrm{Ar}-\mathrm{H}, J=7.7 \mathrm{~Hz}), 7.50(\mathrm{~d}, 1 \mathrm{H}, \mathrm{Ar}-\mathrm{H}, J=8.1 \mathrm{~Hz}), 7.34-7.22(\mathrm{~m}, 3 \mathrm{H}, \mathrm{Ar}-\mathrm{H}), 7.08(\mathrm{t}$, $1 \mathrm{H}, \mathrm{Ar}-\mathrm{H}, J=7.7 \mathrm{~Hz}), 7.02-6.93(\mathrm{~m}, 4 \mathrm{H}, \mathrm{Ar}-\mathrm{H}), 5.74(\mathrm{dd}, 1 \mathrm{H}$, pyrazoline ring, $J=11.7,5.1 \mathrm{~Hz}), 3.85(\mathrm{~s}, 3 \mathrm{H}$, $\left.\mathrm{OCH}_{3}\right), 3.25(\mathrm{dd}, 1 \mathrm{H}$, pyrazoline ring, $J=17.2,5.1 \mathrm{~Hz}$ ), (one of the proton peak of the pyrazoline ring was under methoxy peak). ${ }^{13} \mathrm{C}$ NMR $\left(100 \mathrm{MHz}, \mathrm{CDCl}_{3}, \delta \mathrm{ppm}\right): 163.5,162.5\left({ }^{1} \mathrm{~J}_{\mathrm{CF}}=245 \mathrm{~Hz}\right), 161.5,152.8\left({ }^{2} \mathrm{~J}_{\mathrm{CF}}=22.7\right.$ Hz), 131.9, 128.4, 128.1, 127.9, 125.9, 123.9, 121.9, 120.9, 120.2, 116.1, 115.9, 114.4, 63.1, 55.6, 44.0, HRMS (ESIMS) calc. for $\mathrm{C}_{23} \mathrm{H}_{18} \mathrm{~N}_{3} \mathrm{OFS}[\mathrm{M}+\mathrm{H}]^{+}$404.1227; found 404.1235. 


\section{2-(5-(4-Bromophenyl)-3-(4-methoxyphenyl)-4,5-dihydro-1H-pyrazol-1-yl)benzo[d] thiazole (5)}

A cream colour solid, yield 7.3\%. Mp: $184-185^{\circ} \mathrm{C} .{ }^{1} \mathrm{H}$ NMR $\left(400 \mathrm{MHz}, \mathrm{CDCl}_{3}, \delta \mathrm{ppm}\right): 7.69$ (d, 2H, Ar$\mathrm{H}, J=8.8 \mathrm{~Hz}), 7.64(\mathrm{~d}, 1 \mathrm{H}, \mathrm{Ar}-\mathrm{H}, J=7.7 \mathrm{~Hz}), 7.50(\mathrm{~d}, 1 \mathrm{H}, \mathrm{Ar}-\mathrm{H}, J=8.1 \mathrm{~Hz}), 7.44(\mathrm{~d}, 2 \mathrm{H}, \mathrm{Ar}-\mathrm{H}, J=8.1 \mathrm{~Hz}), 7.27-$ $7.21(\mathrm{~m}, 3 \mathrm{H}, \mathrm{Ar}-\mathrm{H}), 7.08(\mathrm{t}, 1 \mathrm{H}, \mathrm{Ar}-\mathrm{H}, J=7.7 \mathrm{~Hz}), 6.94(\mathrm{~d}, 2 \mathrm{H}, \mathrm{Ar}-\mathrm{H}, J=8.8 \mathrm{~Hz}), 5.70(\mathrm{dd}, 1 \mathrm{H}$, pyrazoline ring, $J=12.1,5.5 \mathrm{~Hz}), 3.85\left(\mathrm{~s}, 3 \mathrm{H}, \mathrm{OCH}_{3}\right), 3.22(\mathrm{dd}, 1 \mathrm{H}$, pyrazoline ring, $J=17.6,5.5 \mathrm{~Hz}$ ), (one of the proton peak of the pyrazoline ring was under methoxy peak). ${ }^{13} \mathrm{C} \mathrm{NMR}\left(100 \mathrm{MHz}, \mathrm{CDCl}_{3}, \delta \mathrm{ppm}\right): 163.5,161.5,152.8,152.6$, 140.7, 132.2, 131.9, 128.4, 128.1, 125.9, 123.9, 122.0, 121.8, 121.0, 120.2, 114.4, 63.2, 55.6, 43.9, HRMS (ESI-MS) calc. for $\mathrm{C}_{23} \mathrm{H}_{18} \mathrm{~N}_{3} \mathrm{OSBr}[\mathrm{M}+\mathrm{H}]^{+}$464.0427; found 464.0421.

\section{2-(5-(3-Bromophenyl)-3-(4-methoxyphenyl)-4,5-dihydro-1H-pyrazol-1-yl)benzo[d] thiazole (6)}

A cream colour solid, yield 13.7\%. Mp: 200-201 ${ }^{\circ} \mathrm{C} .{ }^{1} \mathrm{H}$ NMR (400 MHz, $\left.\mathrm{CDCl}_{3}, \delta \mathrm{ppm}\right): 7.70$ (d, 2H, Ar$\mathrm{H}, J=8.8 \mathrm{~Hz}), 7.64(\mathrm{~d}, 1 \mathrm{H}, \mathrm{Ar}-\mathrm{H}, J=7.7 \mathrm{~Hz}), 7.53-7.51(\mathrm{~m}, 2 \mathrm{H}, \mathrm{Ar}-\mathrm{H})$, 7.40-7.37 (m, 1H, Ar-H), 7.27-7.16 (m, 2H, Ar-H), $7.09(\mathrm{t}, 3 \mathrm{H}, \mathrm{Ar}-\mathrm{H}, J=8.0 \mathrm{~Hz}), 6.94(\mathrm{~d}, 1 \mathrm{H}, \mathrm{Ar}-\mathrm{H}, J=8.8 \mathrm{~Hz}), 5.71(\mathrm{dd}, 1 \mathrm{H}$, pyrazoline ring, $J=12.1$, $5.5 \mathrm{~Hz}), 3.85\left(\mathrm{~s}, 3 \mathrm{H}, \mathrm{OCH}_{3}\right), 3.24(\mathrm{dd}, 1 \mathrm{H}$, pyrazoline ring, $J=17.6,5.5 \mathrm{~Hz})$, (one of the proton peak of the pyrazoline ring was under methoxy peak). ${ }^{13} \mathrm{C} \mathrm{NMR}\left(100 \mathrm{MHz}, \mathrm{CDCl}_{3}, \delta \mathrm{ppm}\right): 163.5,161.5,152.8,152.6$, 143.9, 132.0, 131.1, 130.7, 129.4, 128.4, 125.9, 124.9, 123.9, 123.1, 122.0, 121.0, 120.3, 114.4, 63.2, 55.6, 43.9, HRMS (ESI-MS) calc. for $\mathrm{C}_{23} \mathrm{H}_{18} \mathrm{~N}_{3} \mathrm{OSBr}[\mathrm{M}+\mathrm{H}]^{+}$464.0427; found 464.0412.

\section{2-(5-(2-Bromophenyl)-3-(4-methoxyphenyl)-4,5-dihydro-1H-pyrazol-1-yl)benzo[d] thiazole (7)}

A cream colour solid, yield 18.5\%. Mp: $219-221{ }^{\circ} \mathrm{C} .{ }^{1} \mathrm{H}$ NMR $\left(400 \mathrm{MHz}, \mathrm{CDCl}_{3}, \delta \mathrm{ppm}\right):$ 7.71-7.62 (m, 4H, Ar-H), 7.51 (d, 1H, Ar-H, J = 8.1 Hz), 7.27-7.08 (m, 5H, Ar-H), 6.93 (d, 2H, Ar-H, J = 8.4 Hz), 6.03 (dd, 1H, pyrazoline ring, $J=11.7,5.3 \mathrm{~Hz}), 4.01(\mathrm{dd}, 1 \mathrm{H}$, pyrazoline ring, $J=17.2,11.7 \mathrm{~Hz}), 3.83\left(\mathrm{~s}, 3 \mathrm{H}, \mathrm{OCH}_{3}\right), 3.15(\mathrm{dd}$, $1 \mathrm{H}$, pyrazoline ring, $J=17.6,5.3 \mathrm{~Hz}) .{ }^{13} \mathrm{C} \mathrm{NMR}\left(100 \mathrm{MHz}, \mathrm{CDCl}_{3}, \delta \mathrm{ppm}\right): 163.3,161.5,152.95,152.91,140.2$, 133.5, 132.1, 129.3, 128.4, 128.1, 127.0, 125.9, 123.9, 122.04, 122.0, 121.0, 120.4, 114.4, 63.6, 55.6, 43.2, HRMS (ESI-MS) calc. for $\mathrm{C}_{23} \mathrm{H}_{18} \mathrm{~N}_{3} \mathrm{OSBr}[\mathrm{M}+\mathrm{H}]^{+} 464.0427$; found 464.0430.

\section{2-(5-(2-Chlorophenyl)-3-(4-methoxyphenyl)-4,5-dihydro-1H-pyrazol-1-yl)benzo[d]thiazole (8)}

A bright yellow solid, yield 18.2\%. Mp: 184- $185{ }^{\circ} \mathrm{C} .{ }^{1} \mathrm{H}$ NMR $\left(400 \mathrm{MHz}, \mathrm{CDCl}_{3}, \delta\right.$ ppm): 7.70 (d, 2H, Ar-H, J = 8.8 Hz), $7.64(\mathrm{~d}, 1 \mathrm{H}, \mathrm{Ar}-\mathrm{H}, J=7.3 \mathrm{~Hz}), 7.51(\mathrm{~d}, 1 \mathrm{H}, \mathrm{Ar}-\mathrm{H}, J=8.1 \mathrm{~Hz}), 7.35-7.22(\mathrm{~m}, 5 \mathrm{H}, \mathrm{Ar}-\mathrm{H}), 7.09$ $(\mathrm{t}, 1 \mathrm{H}, J=8.3 \mathrm{~Hz}), 6.94(\mathrm{~d}, 2 \mathrm{H}, \mathrm{Ar}-\mathrm{H}, J=8.8 \mathrm{~Hz}), 5.72(\mathrm{dd}, 1 \mathrm{H}$, pyrazoline ring, $J=11.7,5.1 \mathrm{~Hz}), 3.85(\mathrm{~s}, 3 \mathrm{H}$, $\left.\mathrm{OCH}_{3}\right), 3.24(\mathrm{dd}, 1 \mathrm{H}$, pyrazoline ring, $J=17.2,5.5 \mathrm{~Hz}$ ), (one of the proton peak of the pyrazoline ring was under methoxy peak). ${ }^{13} \mathrm{C}$ NMR (100 MHz, $\left.\mathrm{CDCl}_{3}, \delta \mathrm{ppm}\right): 163.5,161.5,152.8,152.6,143.7,134.9,132.0,130.4$, 128.4, 128.2, 126.5, 125.9, 124.4, 123.9, 122.0, 121.0, 120.2, 114.4, 63.2, 55.6, 43.9, HRMS (ESI-MS) calc. for $\mathrm{C}_{23} \mathrm{H}_{18} \mathrm{~N}_{3} \mathrm{OSCl}[\mathrm{M}+\mathrm{H}]^{+}$420.0932; found 420.0935 .

\section{2-(5-(2,6-Dichlorophenyl)-3-(4-methoxyphenyl)-4,5-dihydro-1H-pyrazol-1-yl)benzo[d]thiazole (9)}

A white solid, yield $27 \%$. Mp: 286-287 ${ }^{\circ} \mathrm{C} .{ }^{1} \mathrm{H}$ NMR $\left(400 \mathrm{MHz}, \mathrm{CDCl}_{3}, \delta\right.$ ppm): 7.74 (d, 2H, Ar-H, J = $8.8 \mathrm{~Hz}), 7.60(\mathrm{~d}, 1 \mathrm{H}, \mathrm{Ar}-\mathrm{H}, J=7.7 \mathrm{~Hz}), 7.42(\mathrm{~d}, 1 \mathrm{H}, \mathrm{Ar}-\mathrm{H}, J=7.7 \mathrm{~Hz}), 7.26-7.14(\mathrm{~m}, 4 \mathrm{H}, \mathrm{Ar}-\mathrm{H}), 7.04(\mathrm{t}, 1 \mathrm{H}, \mathrm{Ar}-$ $\mathrm{H}, J=7.5 \mathrm{~Hz}), 6.96(\mathrm{~d}, 2 \mathrm{H}, \mathrm{Ar}-\mathrm{H}, J=8.8 \mathrm{~Hz}), 6.40(\mathrm{dd}, 1 \mathrm{H}$, pyrazoline ring, $J=20.5,8.8 \mathrm{~Hz}), 3.86(\mathrm{~s}, 3 \mathrm{H}$, $\left.\mathrm{OCH}_{3}\right), 3.46(\mathrm{dd}, 1 \mathrm{H}$, pyrazoline ring, $\mathrm{J}=17.2,8.8 \mathrm{~Hz}$ ), (one of the proton peak of the pyrazoline ring was under methoxy peak). HRMS (ESI-MS) calc. for $\mathrm{C}_{23} \mathrm{H}_{17} \mathrm{~N}_{3} \mathrm{OSCl}_{2}[\mathrm{M}+\mathrm{H}]^{+} 454.0542$; found 454.0547 .

\subsection{Acetylcholinesterase inhibitory assay}

The effects of compounds on AChE enzyme were investigated. Two substrates which are 5,5dithiobis(2-nitrobenzoic acid) (DTNB) and acetylcholiniodate (AChI) were used in the inhibition tests. In addition, Tris / $\mathrm{HCl}(100 \mathrm{ml}, 1 \mathrm{M}, \mathrm{pH}=8)$ buffer was used in this method for AChE enzyme. The final volume was completed to $100 \mu \mathrm{L}$ and changes in the absorption were followed at $412 \mathrm{~nm}$. The IC $_{50}$ values of the compounds were calculated via activity (\%) versus compounds concentration plots [33].

Acknowledgements: The authors thank to TUBITAK for financial support (Project Number: 117S939) and Ataturk University (Erzurum, Turkey) for NMR spectra.

Author contributions: Concept - M.T., H.I.G.; Design - M.T., H.I.G.; Supervision - H.I.G.; Resources - M.T., H.I.G.; I.G; Materials - M.T.; Data Collection and/or Processing -M.T.; Analysis and/or Interpretation - H.I.G., M.T., I.G.; Literature Search - M.T.; Writing - M.T.; H.I.G.; I.G; Critical Reviews - H.I.G., M.T., I.G

Conflict of interest statement: The authors declared no conflict of interest" in the manuscript. 


\section{REFERENCES}

[1] Ragab HM, Teleb M, Haidar HR, Gouda N. Chlorinated tacrine analogs: design, synthesis and biological evaluation of their anti-cholinesterase activity as potential treatment for Alzheimer's disease. Bioorg Chem. 2019; 86: 557-568. [CrossRef]

[2] Kumar B, Dwivedi AR, Sarkar B, Gupta SK, Krishnamurthy S, Mantha AK, Parkash J, Kumar V. 4,6Diphenylpyrimidine derivatives as dual inhibitors of monoamine oxidase and acetylcholinesterase for the treatment of Alzheimer's disease. Acs Chem Neurosci. 2019; 10(1): 252-265. [CrossRef]

[3] Carmo Carreiras M, Ismaili L, Marco-Contelles J. Propargylamine-derived multi-target directed ligands for Alzheimer's disease therapy. Bioorg Med Chem Lett. 2020; 30(3): 126880. [CrossRef]

[4] Zhou A, Hu JP, Wang LR, Zhong GC, Pan J, Wu ZY, Hui A. Combined 3D-QSAR, molecular docking, and molecular dynamics study of tacrine derivatives as potential acetylcholinesterase (AChE) inhibitors of Alzheimer's disease. J Mol Model. 2015; 21(10): 277. [CrossRef]

[5] Carter MD, Simms GA, Weaver DF. The development of new therapeutics for Alzheimer's disease. Clin Pharmacol Ther. 2010; 88(4): 475-486. [CrossRef]

[6] Sasikala R, Thirumurthy K, Mayavel P, Thirunarayanan G. Eco-friendly synthesis and antimicrobial activities of some 1-phenyl-3(5-bromothiophen-2-yl)-5-(substituted phenyl)-2-pyrazolines. Org Med Chem Lett. 2012; 2(1): 20. [CrossRef]

[7] Chen L, Duan H, Zhang X, Zhang Q, Huang H, Zhao J, Chen B, Hua C, Gou X. Design, synthesis, and biological evaluation of novel 1,3,4- thiadiazolylpyrazolines compounds containing ferrocene. J Heterocyclic Chem. 2018; 55, 1978. [CrossRef]

[8] Kumar G, Tanwar O, Kumar J, Akhter M, Sharma S, Pillai CR, Alam MM, Zaman MS. Pyrazole-pyrazoline as promising novel antimalarial agents: a mechanistic study. Eur J Med Chem. 2018; 149: 139-147. [CrossRef]

[9] Eid NM, George RF. Facile synthesis of some pyrazoline-based compounds with promising anti-inflammatory activity. Future Med Chem. 2018; 10(2): 183-199. [CrossRef]

[10] Gokhan-Kelekci N, Yabanoglu S, Kupeli E, Salgin Goksen U, Ozgen O, Ucar G, Yesilada E, Kendi E, Yesilada A, Bilgin AA. A new therapeutic approach in Alzheimer disease: some novel pyrazole derivatives as dual MAO-B inhibitors and antiinflammatory analgesics. Bioorg Med Chem. 2007; 15(17): 5775-5786. [CrossRef]

[11] Shah MS, Khan SU, Ejaz SA, Afridi S, Rizvi SUF, Najam-Ul-Haq M, Iqbal J. Cholinesterases inhibition and molecular modeling studies of piperidyl-thienyl and 2-pyrazoline derivatives of chalcones. Biochem Biophys Res Commun. 2017; 482(4): 615-624. [CrossRef]

[12] Ucar G, Gokhan N, Yesilada A, Bilgin AA. 1-N-Substituted thiocarbamoyl-3-phenyl-5-thienyl-2-pyrazolines: a novel cholinesterase and selective monoamine oxidase B inhibitors for the treatment of Parkinson's and Alzheimer's diseases. Neurosci Lett. 2005; 382(3): 327-331. [CrossRef]

[13] Can OD, Ozkay UD, Kaplancikli ZA, Ozturk Y. Effects of some 1,3,5-trisubstitued-2-pyrazoline derivatives on depression and anxiety parameters of mice. Arch Pharm Res. 2009; 32(9): 1293-1299. [CrossRef]

[14] Ozdemir A, Altintop MD, Kaplancikli ZA, Can OD, Ozkay UD, Turan-Zitouni G. Synthesis and evaluation of new 1,5-diaryl-3-[4-(methyl-sulfonyl)phenyl]-4,5-dihydro- $1 H$-pyrazole derivatives as potential antidepressant agents. Molecules. 2015; 20(2): 2668-2684. [CrossRef]

[15] Gomha SM, Abdallah MA, Abbas IM, Kazem MSH. Synthesis, cytotoxicity evaluation, molecular docking and utility of novel chalcones as precursors for heterocycles incorporating pyrazole moiety. Med Chem. 2018; 14(4): 344355.

[16] Gul HI, Yamali C, Sakagami H, Angeli A, Leitans J, Kazaks A, Tars K, Ozgun DO, Supuran CT. New anticancer drug candidates sulfonamides as selective hCA IX or hCA XII inhibitors. Bioorg Chem. 2018; 77: 411-419. [CrossRef]

[17] Chimenti F, Bolasco A, Manna F, Secci D, Chimenti P, Granese A, Befani O, Turini P, Cirilli R, La Torre F, Alcaro S, Ortuso F, Langer T. Synthesis, biological evaluation and 3D-QSAR of 1,3,5-trisubstituted-4,5-dihydro-(1H)-pyrazole derivatives as potent and highly selective monoamine oxidase a inhibitors. Curr Med Chem. 2006; 13(12): 14111428 .

[18] Jagrat M, Behera J, Yabanoglu S, Ercan A, Ucar G, Sinha BN, Sankaran V, Basu A, Jayaprakash V. Pyrazoline based MAO inhibitors: synthesis, biological evaluation and SAR studies. Bioorg Med Chem Lett. 2011; 21(14): 4296-4300. [CrossRef] 
[19] Ozgun DO, Gul HI, Yamali C, Sakagami H, Gulcin I, Sukuroglu M, Supuran CT. Synthesis and bioactivities of pyrazoline benzensulfonamides as carbonic anhydrase and acetylcholinesterase inhibitors with low cytotoxicity. Bioorg Chem. 2019; 84: 511-517. [CrossRef]

[20] Yamali C, Gul HI, Ece A, Taslimi P, Gulcin I. Synthesis, molecular modeling, and biological evaluation of 4-[5-aryl3-(thiophen-2-yl)-4,5-dihydro-1H-pyrazol-1-yl] benzenesulfonamides toward acetylcholinesterase, carbonic anhydrase I and II enzymes. Chem Biol Drug Des. 2018; 91(4): 854-866. [CrossRef]

[21] Gul HI, Tugrak M, Sakagami H, Taslimi P, Gulcin I, Supuran CT. Synthesis and bioactivity studies on new 4-(3-(4substitutedphenyl)-3a,4-dihydro-3H-indeno[1,2-c]pyrazol-2-yl) benzenesulfonamides. J Enzyme Inhib Med Chem. 2016; 31(6): 1619-1624. [CrossRef]

[22] Kucukbay H, Durmaz B. Antifungal activity of organic and organometallic derivatives of benzimidazole and benzothiazole. Arzneimittelforsch. 1997; 47(5): 667-670.

[23] Kalina PH, Shetlar DJ, Lewis RA, Kullerstrand LJ, Brubaker RF. 6-Amino-2-benzothiazole-sulfonamide. The effect of a topical carbonic anhydrase inhibitor on aqueous humor formation in the normal human eye. Ophthalmol. 1988; 95(6): 772-777. [CrossRef]

[24] Mohr E, Nair NP, Sampson M, Murtha S, Belanger G, Pappas B, Mendis T. Treatment of Alzheimer's disease with sabeluzole: functional and structural correlates. Clin Neuropharmacol. 1997; 20(4): 338-345.

[25] Kagaya T, Kajiwara A, Nagato S, Akasaka K, Kubota A. E2011 a novel, selective and reversible inhibitor of monoamine oxidase type A. J Pharmacol Exp Ther. 1996; 278(1): 243-251.

[26] Aboelmagd A, Ali IAI, Salem EMS, Abdel-Razik M. Synthesis and antifungal activity of some smercaptotriazolobenzothiazolyl amino acid derivatives. Eur J Med Chem. 2013; 60: 503-511. [CrossRef]

[27] Gul HI, Tugrak M, Gul M, Mazlumoglu S, Sakagami H, Gulcin I, Supuran CT. New phenolic mannich bases with piperazines and their bioactivities. Bioorg Chem. 2019; 90: 103057. [CrossRef]

[28] Gul HI, Tugrak M, Gul M, Sakagami H, Umemura N, Anil B. Synthesis and cytotoxicities of new azafluorenones with apoptotic mechanism of action and cell cycle analysis. Anticancer Agents Med Chem. 2018; 18(12): 1770-1778.

[29] Tugrak M, Gul HI, Bandow K, Sakagami H, Gulcin I, Ozkay Y, Supuran CT. Synthesis and biological evaluation of some new mono mannich bases with piperazines as possible anticancer agents and carbonic anhydrase inhibitors. Bioorg Chem. 2019; 90: 103095. [CrossRef]

[30] Tugrak M, Gul HI, Sakagami H, Gulcin I, Supuran CT. New azafluorenones with cytotoxic and carbonic anhydrase inhibitory properties: 2-aryl-4-(4-hydroxyphenyl)-5H-indeno[1,2-b]pyridin-5-ones. Bioorg Chem. 2018; 81: 433-439. [CrossRef]

[31] Tugrak M, Yamali C, Sakagami H, Gul HI. Synthesis of mono mannich bases of 2-(4-hydroxybenzylidene)-2,3dihydroinden-1-one and evaluation of their cytotoxicities. J Enzyme Inhib Med Chem. 2016; 31(5): 818-823. [CrossRef]

[32] Sharma V, Sharma KV. Synthesis and biological activity of some 3,5-diaryl-1-benzothiazolopyrazoline derivatives: reaction of chalcones with 2-hyrazinobenzothiazoles. E-J Chem. 2009; 6(2): 348-356. [CrossRef]

[33] El-Sayed NA, Farag AE, Ezzat MAF, Akincioglu H, Gulcin I, Abou-Seri SM. Design, synthesis, in vitro and in vivo evaluation of novel pyrrolizine-based compounds with potential activity as cholinesterase inhibitors and antiAlzheimer's agents. Bioorg Chem. 2019; 93: 103312. [CrossRef] 